\title{
WAYS OF SWITCHING OF STATIC MAGNETIC FIELD AND ITS INFLUENCE OVER THE WORK PARAMETERS OF MAGNETIC CIRCUITS
}

\author{
Ventsislav Keseev ${ }^{1}$ \\ ${ }^{1}$ Telecommunications department, University of Ruse “A. Kanchev”, Ruse, Bulgaria
}

\begin{abstract}
There are many patents claiming that static magnetic field of permanent magnet could be electronically redirected under certain circumstances and useful work could be achieved. The way of work of those devices is very close to that of a flyback transformer but with incorporated permanent magnet in it. No any science information have been found regarding what's the influence of a magnet incorporated into the structure of a transformer, particularly a flyback transformer, over its work parameters. The paper covers preliminary examination of the effects of such incorporation. The investigation aims to clarify how those effects change with different air gap lengths and what would be the final result concerning the overall transformer behavior. The paper also presents a theory concerning possible ways for manipulation of static magnetic field and final result expectations.
\end{abstract}

Keywords: permanent magnet, flyback transformer, static magnetic field, air gap

\section{INTRODUCTION}

Magnets are all around us nowadays. People are so used to them that only few ask where their force comes from. If certain magnetic force is needed for some kind of work we could make an electromagnet which will consume energy in order to magnetize a core. As soon as energy is being consumed the force will be there but if the power is switched off then it will disappear. It isn't so with magnets. Their force is static and it is there for long periods of time. Magnets are everywhere and they do work without consuming any energy at all. They could hold a heavy souvenir on the fridge for years not requiring even a drop of extra energy and this is accepted as something normal. If the existence of relatively static magnetic force is possible and it is real then there should be ways that force to be used under some special conditions for useful work and why not for energy generation. It is well known that if a wire is placed in changing magnetic field then electromagnetic force is induced. If there is a way the static magnetic field of a strong magnet to be deflected with low-power force to flow in different pathways then with the help of solenoids energy could be extracted out of the system which will be possible thanks to the artificially created changing magnetic field. Many patents state that static magnetic field could be driven under some special conditions $[8-14,17,18,19]$. There are also designs of magnetic switches which are claimed to be able to operate in a similar manner as electronic switches which representatives are the transistors. Transistors make it possible energy from high-power sources to be switched with considerably lower power. If there are such electric switches there should be way similar magnetic ones to be created. There are some patents presenting different designs of magnetic switch devices pretending that the effect of switching of static magnetic field is actually possible but there isn't a lot of information pointing out how much drive energy is needed.

The decision for this investigation has been taken after patent research which revealed many patents claiming that static magnetic field could be manipulated and useful work could be achieved. Some newer patents even claim the possibility of extra energy generation under certain circumstances thanks to the static magnetic force artificial switching. At the same time science paper research didn't show any clear information concerning what change does a magnet incorporated into the ferrite core brings. The lack of clear science information on this matter summed with the patent claims pointing out that certain effects exist brings the necessity of this research. The aim of this examination is to reveal how magnet incorporation into a core influences the overall transformer work and to check the possibility of existence of certain effects which could lead to the claimed results. It is well known that many effects claimed to be low significant or parasitic in some circumstances under others turn to be helpful and useful in modern technology. For more precise results information of how much certain magnet magnetizes the core is necessary. These preliminary experiments intend to gather that information which will be used in future research.

\section{EXPERIMENTAL SETUP}

Measurements of how much different magnets magnetize a ferrite core with different air gaps will be completed using the setup shown on fig. 1 . 


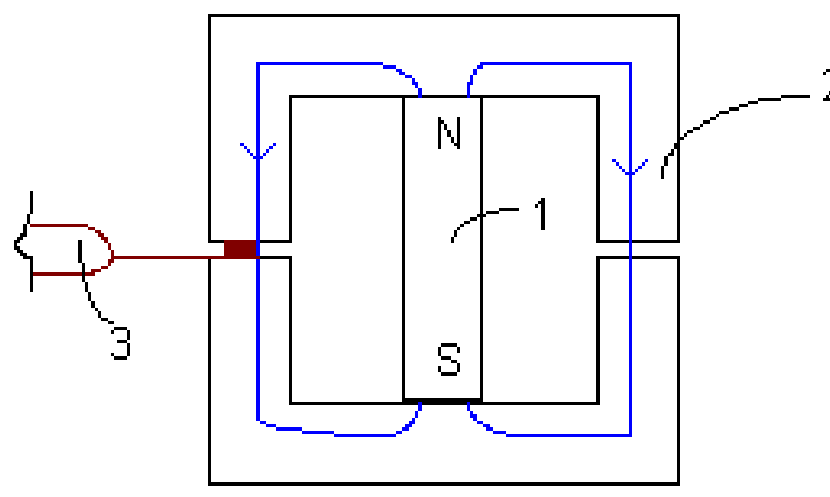

(a)

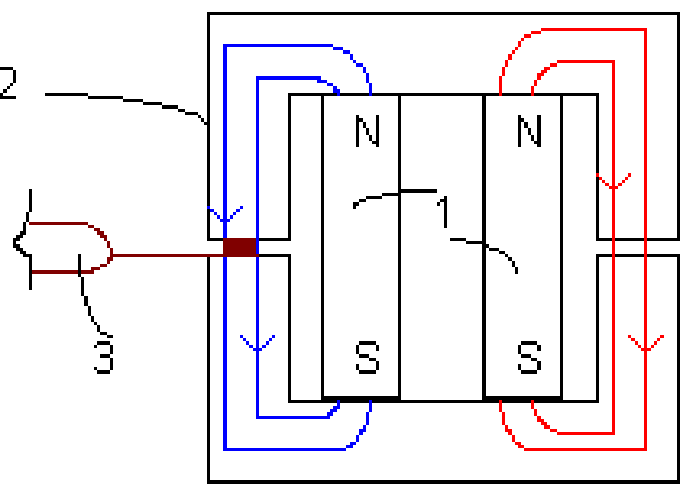

(b)

Fig 1 Measurements setup: (a) Setup with only one magnet stack; (b) Setup with two magnet stacks; 1-magnets; 2-ferrite core; 3teslameter probe

Level of magnetization of the core could be measured only if the magnet is positioned vertically as in fig. 1. This way magnetic force lines will go directly through the air gap and the measurement probe. If the magnets were placed horizontally then there won't be magnetic force lines crossing the air gap and the Teslameter would show 0 .
Magnets with equal two dimensions and different thickness will be stacked up until the necessary height is achieved such one which will allow for different air gaps. The number of magnets with different sizes are limited so 2 different air gaps would be possible.

\subsection{Selecting a Ferromagnetic Core}

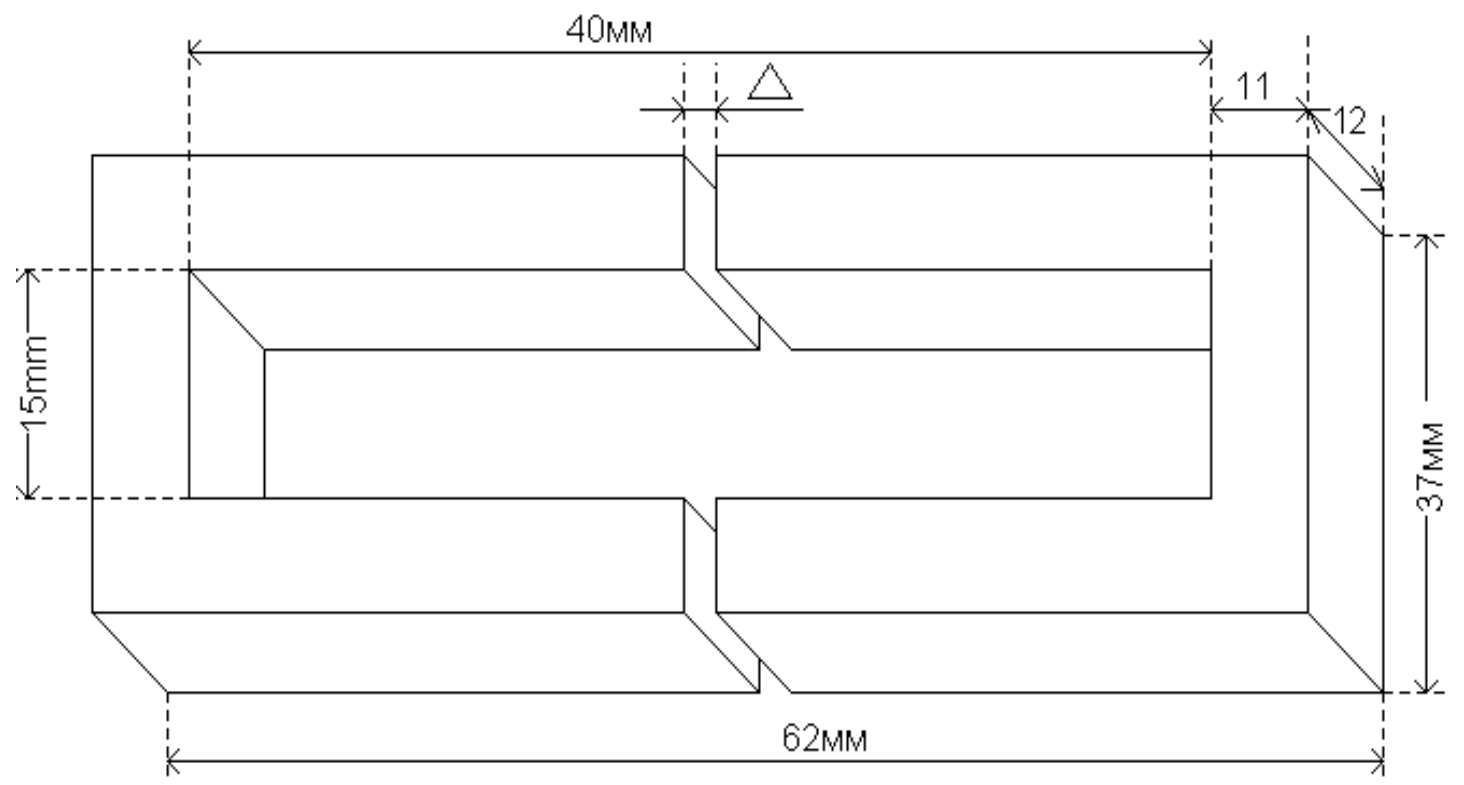

Fig 2 Dimensions of the ferrite core

It is very difficult to find an existing size ferrite core which will allow the incorporation of many magnets with the same height but different shape and force. At the same time the core should have large enough window which will allow for primary and secondary windings. A core from an old flyback transformer is found to be good for matching with certain sizes existing magnets fig. 2 . The size of $15 \mathrm{~mm}$ will be matched with stacks of magnets with the same height and different other dimensions. The core has an effective cross sectional area $A_{e}=130 \mathrm{~mm}^{2}$.

\subsection{Selecting Magnets}

Ferrite and Neodymium magnets with different sizes could be used. The ferrite ones turned out to be too weak so they won't be used. The dimensions and forms of available Neodymium magnets are shown on fig.3. All magnets are magnetized along the $15 \mathrm{~mm}$ dimension. Their magnetic flux density $\mathrm{B}_{\mathrm{M}}$ measured with Teslameter is in table 1 . 
Table 1 Magnetic flux density measurements

\begin{tabular}{|c|c|c|c|c|c|c|c|}
\hline \multirow{2}{*}{$\begin{array}{l}\text { Magnet } \\
\text { dimensio } \\
\mathrm{ns}(\mathrm{mm})\end{array}$} & \multirow{2}{*}{$\begin{array}{l}\text { Number } \\
\text { of magnet } \\
\text { stacks }\end{array}$} & \multirow{2}{*}{$\begin{array}{l}\text { Magnet } \\
\text { cross } \\
\text { sectional } \\
\text { area } \\
\mathrm{S}_{\mathrm{M}}\left(\mathrm{mm}^{2}\right)\end{array}$} & \multirow{2}{*}{$\begin{array}{l}\text { Magnet } \\
\text { flux } \\
\text { density } \\
\mathrm{B}_{\mathrm{M}}(\mathrm{mT})\end{array}$} & \multirow{2}{*}{$\begin{array}{l}\text { Core to } \\
\text { magnet } \\
\text { surface ratio } \\
2 \mathrm{~A}_{\mathrm{e}} / \mathrm{S}_{\mathrm{M}}\end{array}$} & \multirow{2}{*}{$\begin{array}{l}\text { Calculated } \\
\text { core flux } \\
\text { density } \\
\mathrm{B}_{\mathrm{C}}(\mathrm{mT})\end{array}$} & \multicolumn{2}{|c|}{$\begin{array}{l}\text { Measured core flux } \\
\text { density } B_{R}(m T)\end{array}$} \\
\hline & & & & & & $\begin{array}{l}\text { Air gap } \\
\text { width } \\
\Delta=4 \mathrm{MM}\end{array}$ & $\begin{array}{l}\text { Air gap } \\
\text { width } \\
\Delta=10 \mathrm{Mm}\end{array}$ \\
\hline$\Phi 5 \times 15$ & 1 & 19.6 & 450 & 13.27 & 34 & 28 & 17 \\
\hline $\begin{array}{l}5 \times 10 \times \\
15\end{array}$ & 1 & 50 & 550 & 5.20 & 106 & 88 & 50 \\
\hline $\begin{array}{l}\Phi 10 \quad \mathrm{x} \\
15\end{array}$ & 1 & 78.5 & 550 & 3.31 & 167 & 140 & 80 \\
\hline $\begin{array}{l}\Phi 10 \quad \mathrm{x} \\
15\end{array}$ & 2 & 157.1 & 550 & 1.66 & 331 & 276 & 161 \\
\hline
\end{tabular}
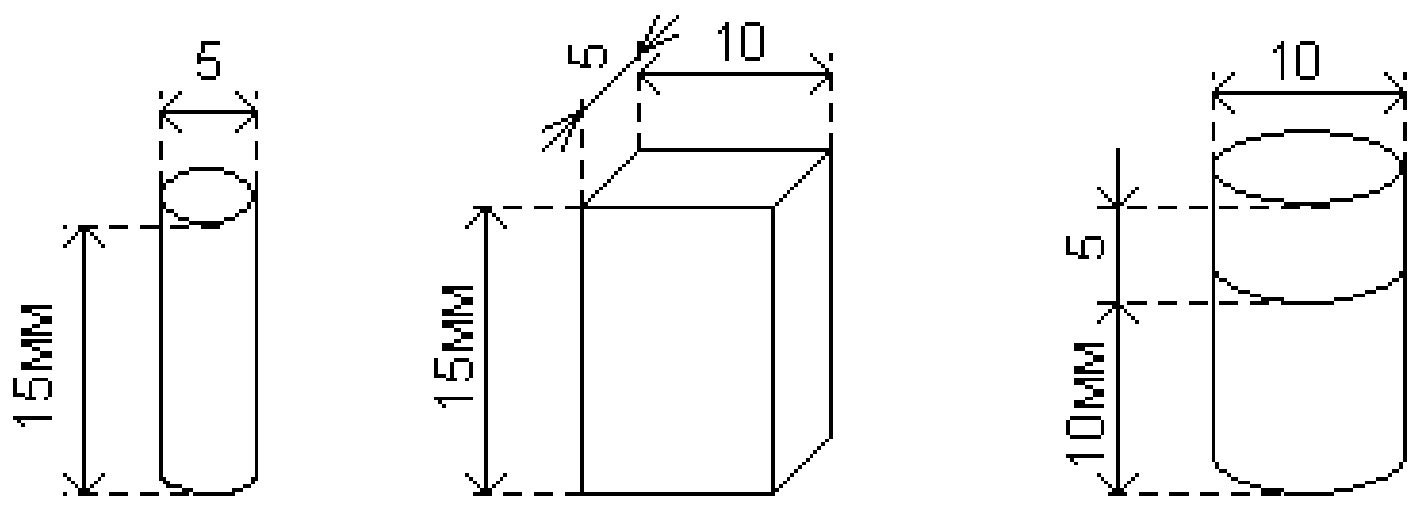

Fig 3 Dimensions of the magnets

\subsection{Selecting a Magnetic Induction Meter}

Conclusions of how strong the magnets are and how magnetized the core is will be made based on measurements with a Teslameter model HT20 which could measure magnetic field densities up to 2 tesla. The meter probe thickness is $1.5 \mathrm{~mm}$ which will allow for measurements with air gap longer than that.

\section{MEASUREMENT RESULTES}

\subsection{Core Magnetic Flux Density Measurements}

All of the experimental results are in table 1. The magnets cross sectional areas are calculated using the geometry formulas for area of a circle if they are cylindrical or area of rectangle if their shape is rectangular $(1,2)$. Their core flux densities have been measured at the middle of their cross sectional areas.

$$
\begin{aligned}
& S_{M}=\pi r^{2} \\
& S_{M}=a \cdot b
\end{aligned}
$$

It is expected that a magnetic flux with certain density springing out of a magnet with given surface area, if it is divided and conducted to flow through two different pathways as shown in fig. 1 (a), then the flux density through the alternative channels should be inversely proportional to the ratio of the sum of the surface areas of both channels towards the surface area of the magnet. This statement means that if for example a magnet has a magnetic flux $\Phi_{M}$ (3), flux density $B_{M}$ and cross sectional area $S_{M}$ and if its stationary magnetic flux is divided to flow trough two different high permeability pathways with summary surface area $2 \mathrm{~A}_{\mathrm{E}}$ four times larger than that of the magnet (4), then the flux density $\mathrm{B}_{\mathrm{C}}$ in any one of the two pathways should be four times lower (5).

$$
\begin{gathered}
\Phi_{M}=B_{M} \times S_{M} \\
A_{E}=2 S_{M} \\
B_{C}=\frac{\Phi_{M}}{2 A_{E}}=\frac{B_{M} \times S_{M}}{4 S_{M}}=\frac{B_{M}}{4}
\end{gathered}
$$

It is well seen that the bigger the air gap the lower measured core flux density, table 1 . This effect is due to the fact that wider air gap means lower total core permeability which leads to more of the static magnetic field force lines flowing through the space instead through the core. Measurements point out that the calculation of the ferrite core magnetic flux density is correct only for the minimal air gap case, the bigger the air gap length the bigger the overshot. The same has been found to be valid for calculations of level of magnetization of gapped ferrite cores based on the magnetic flux density equation (7), [6]. 


\subsection{Inductance Measurements}

Windings of 95 turns have been wound on the core. The total air gap length in this case is $3.5 \mathrm{~mm}$. The are in table 2 . Measurements show that the stronger the magnet incorporated, the lower the inductance. This effect should be due to the partially magnetized core which should be taken under consideration during the design process.

Table 2 Inductance measurements

\begin{tabular}{|l|l|l|l|l|}
\hline Magnets & None & $\begin{array}{l}\Phi 5 \\
15\end{array}$ & $\begin{array}{l}5 \times 10 \\
\text { x 15 }\end{array}$ & $\begin{array}{l}\text { Ф10 x } \\
15\end{array}$ \\
\hline $\begin{array}{l}\text { Inductance } \\
\text { L, mH }\end{array}$ & 1.62 & 1.6 & 1.55 & 1.43 \\
\hline
\end{tabular}

\subsection{Simple Experiment Proving Faraday's Law of}

\section{Induction}

Faraday's law basically states that if a wire or solenoid is being moved across the lines of magnetic field then electromotive force will be induced in it $[1,5]$. This law has been proved to be true. This law means that if the magnetic field of a permanent magnet could be redirected trough two or more different high permeability magnetic pathways and if there are output windings wound around them, then electromotive force should be induced due to the artificially changing magnetic field. The stationary magnetic field redirection could be achieved by changing the permeability of the different pathways. The easiest way is to change the air-gap length, the higher the air-gap length the lower the permeability. If the setup of fig.1 (a) is used, if the permeability of the left branch of the ferrite core becomes higher than that of the right one, then the magnetic force lines of the magnet will redistribute, which means that the branch with higher permeability will have higher magnetic flux density. Then if we switch the permeability of both branches, the right one to be with higher permeability than the left one, we will have the opposite case. Finally the changing levels of magnetization of both halves of the ferrite core are supposed to induce EMF in the output windings. This theory could be verified by leading simple experiment with the help of setup shown on fig. 4.

If the two halves of the ferrite core are being swayed by hand against the magnet this way the air gap length could be varied fast enough from certain minimum to certain maximum, which will lead to alternate change in the permeability of the left and right parts of the core. In this case if we connect Amperemeter or Voltmeter across the output windings which are W1-W2 and W3-W4 connected in series, we could measure if any voltage or current appears there. Each winding has 50 turns so W1 and W2 connected in series would have 100 turns all together.

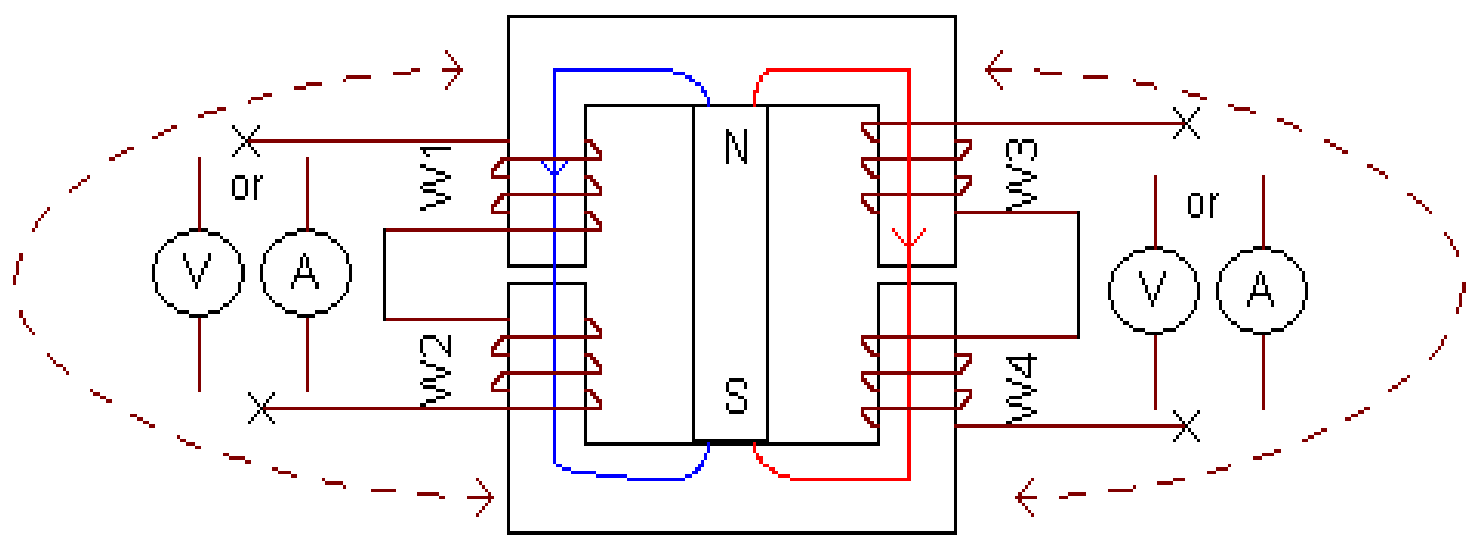

Fig 4 Induction law experimental setup

When the air gap is maximal then the inductance of either output winding $\mathrm{W} 1 \mathrm{~W} 2$ or $\mathrm{W} 3 \mathrm{~W} 4$ is measured to be $\mathrm{L}_{\Delta \max }=713 \mu \mathrm{H}$.

Two experiments are done. The first one is with minimal airgap which is the case when both halves touch each other. In the second case the minimal air-gap length is limited to $4 \mathrm{~mm}$ with the help of separators. The results are shown in table 3 .

The measurements of the AC and DC voltages and currents have been done with multimeter Mastech MS8220R. The values written in table 3 are the maximum ones which the meter shows for the fastest possible moving of the ferrite core halves. In case I compared to case II we have bigger change of the air gap length which leads to about 4 times higher maximum inductance measurement. The same is visible for the measured values of voltages and currents which although small clearly show that there is output energy generation. The output induced EMF is actually due to the successful redirection of the static magnetic field. Another visible conclusion from this experiment is that the higher and faster the air-gap lengths change the higher the induced voltages.

This experiment proves that energy generation based on redirection of static magnetic field of a permanent magnet is possible by changing the magnetic permeability of magnetic path and consecutive redirection of the magnetic flux trough alternative one. The question is: Is it possible the redirection to be done motionlessly? There are many patents stating that this is indeed possible. If motionless redirection is possible, is it possible the field of a strong magnet to be redirected with 
low input power? Scientific paper research didn't show any information concerning that matter. Thorough patent research revealed hat there are old and new patents claiming that they have succeeded in motionless permanent magnet flux redirection and some of them even claim that useful work have been achieved.

Table 3 Experimental results

\begin{tabular}{|l|l|l|l|l|l|l|}
\hline & $\mathrm{L}_{\Delta \max }, \mu \mathrm{H}$ & $\mathrm{L}_{\Delta \min }, \mu \mathrm{H}$ & $\mathrm{U}_{\mathrm{DC} M}, \mathrm{mV}$ & $\mathrm{I}_{\mathrm{DC} M}, \mathrm{~mA}$ & $\mathrm{U}_{\mathrm{AC} M}, \mathrm{mV}$ & $\mathrm{I}_{\mathrm{AC} M}, \mathrm{~mA}$ \\
\hline I case: $\Delta_{\min }=\min$ & 713 & 4300 & 35 & 5 & 50 & 7.5 \\
\hline II case: $\Delta_{\min }=4 \mathrm{~mm}$ & 713 & 1084 & 10 & 1.5 & 15 & 2.2 \\
\hline
\end{tabular}

\subsection{Magnet Lifespan}

Neodymium magnets are said to have lifespan between 10 to 20 years. If they work in an environment with strong magnetic fields their lifespan could be shortened. Guiding the static magnetic flux to flow in one or another direction shouldn't be considered as worst case scenario as guiding the static magnetic field doesn't mean opposing it. Simple experiment has been done. A Neodymium magnet has been stuck to $U$ high permeability ferrite core as shown in fig. 5 for two months. Magnetic flux density measurements show no any considerable difference before and after the experiment. This experiment shows that the magnet doesn't get visibly demagnetized due to the high permeability link between its south and north poles. This simple experiment proves that Neodymium magnets could be incorporated in ferrite cores and they are not supposed to lose their strength faster than normal in time.

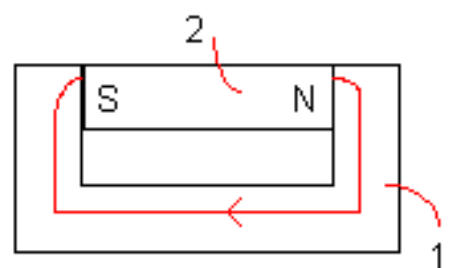

Fig 5 Magnet with high permeability connection between its poles: 1- magnet; 2- ungapped ferrite core

\section{WAYS FOR MOTIONLESS CONTROL OF STATIC MAGNETIC FIELD}

Patent research revealed that there are designs of magnetic flux switches dating from 1900 to 2010. Most of the magnetic switches designs are working based on creation of opposite magnetomotive force or magnetic permeability change. All dose designs could be divided in two groups, the first being switches with moving parts and the secondmotionless switches. It is well known form practice that moving parts are not desired because in most cases they are the week part of the system requiring maintenance.

Most of the motionless switch models are working based on redirection of magnetic flux with the help of control windings. There are other magnetic switch models working based on the magnetostrictive effect, but there is little information about them [17]. There are two a little different ways of redirection of magnetic flux with the help of control windings, the first being called from us direct and the second indirect control. Representatives of both ways of control are displayed on fig. 6 and fig. 7.

\subsection{Direct Control Way}

In the case with direct switching there is only one gapped ferrite core around which are wound the control windings and the output ones. The main idea of control is that if one of the control windings is powered the created electro-magnetic field will interact with that of the magnet and will lead to higher net magnetic flux flowing trough one of the ferrite core halves at the expense of the another. If the other control winding is activated then the process will develop in opposite direction.

The gapped ferrite core 1 and 2, fig.6 together with the symmetrically integrated permanent magnet 3 are parts of a system in balance, where both halves of the core are equally magnetized but in opposite directions. When power is applied to winding W1 par example, the current flowing through its turns will magnetize the whole core one way to certain level- let's say in the counterclockwise direction. In ferromagnetic halve 1 of the core this magnetization will be in line with the permanent magnet force lines and will lead to proportional increase of the magnetic flux density. In halve 2 this magnetization will oppose the permanent magnet field and this will lead to proportional drop of the magnetic flux density in it. At the end it is expected that certain energy consumed will create proportional imbalance in the system. When the applied outside electromagnetic force ceases then the whole system will return to its initial state and then in the ideal case the same amount of energy should be given back, based on the fact that all parameters of the magnetic circuit are the same and haven't changed. In reality there will be losses and the energy will be less.

Energy collected in a solenoid is proportional to half of the product of the inductance $\mathrm{L}$ and the square of the current $\mathrm{I}^{2}$ flowing through the windings (6).

$$
W=\frac{1}{2} I^{2} L
$$

The inductance is proportional to the product of the gapped core magnetic permeability $\mu$, the square of the number of turns $\mathrm{N}^{2}$ and the effective cross section area of the core $\mathrm{A}_{\mathrm{E}}$, divided to the length of a magnetic flux line (7).

$L=\mu \frac{N^{2} A_{E}}{l}$ 


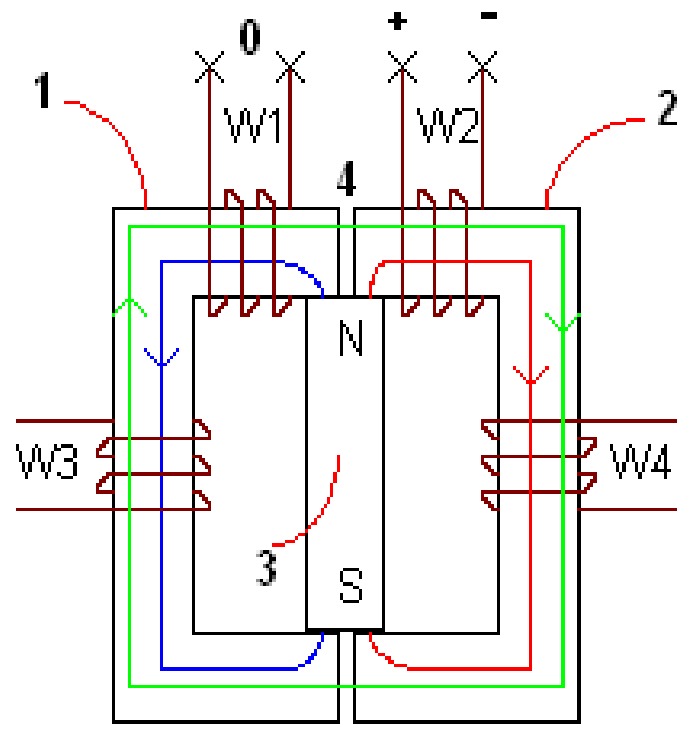

4

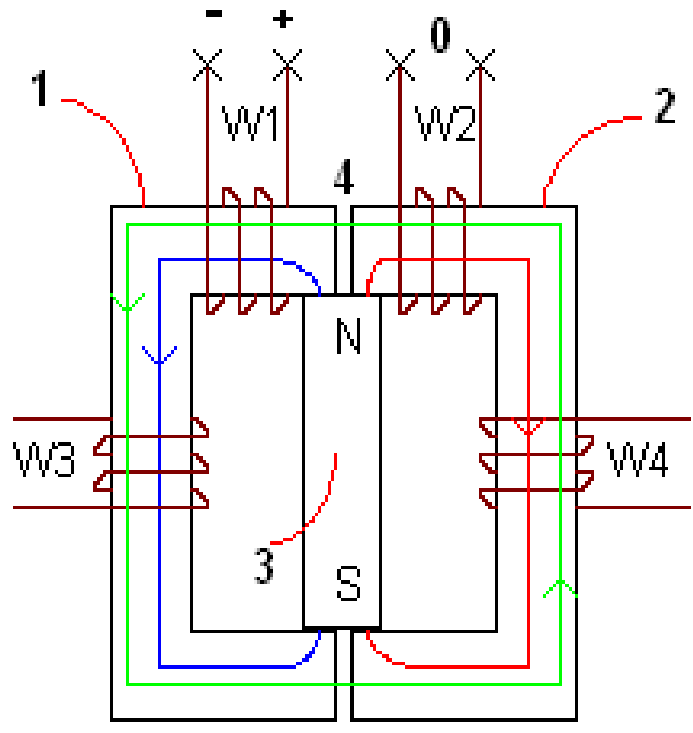

4

Fig 6 Direct control: 1 and 2- left and right ferrite core halves; 3- permanent magnet; 4 - air-gap; W1 and W2 - control windings; W3 and W4 output windings

If inductance equation (7), magnetic flux density equation (8) and (6) are combined then the equation for energy in an inductor reduces to (9).

$$
\begin{aligned}
& B=\mu H=\mu \frac{W I}{l} \\
& W=\frac{A_{E} B^{2} l}{2 \mu}
\end{aligned}
$$

If the core gets magnetized by a permanent magnet this also means that we have entered energy into that system. The question is how much is that energy?

Energy stored in an inductor is proportional to the square of the magnetic flux density in the core and inversely proportional to the core permeability. With the help of equation (9) if we examine certain design and we know the ferrite core parameters and its permeability which depends on the chosen air gap then the energy induced in an output winding while core gets demagnetized could be calculated. If any of the control windings is powered up, then this will lead to certain change of the magnetic flux density and to respective change of the energy collected in the system. When we disconnect the power supply then the system will return to initial state and the magnetic flux density is supposed to return to its initial value. If it returns to the initial value when all other parameters don't change at all, then the same amount of energy will be given back. In reality magnetic circuits are characterized with such called memory effect. It is known that the magnetic flux flowing trough magnetic path with certain permeability refuses to redistribute when other alternative magnetic paths are added to the system. This effect is not in help of the working principles described herein.
Based on the theory the direct way of control of strong magnetic field with low drive power seems to be impossible, yet there are patents claiming that working models have been developed. Often patent holders omit important details about their inventions with the clear aim others not to be able to recreate them. Whether this is the case or not, only practical examination will prove.

\subsection{Indirect Control Way}

Representative model of the indirect switching way is displayed on fig.7. This way is different with the fact that the control windings 3 are wound around their own highpermeability ferrite core which has a common section with the main magnetic circuit being gapped ferrite core consisted of halves 1 and 2 around which the output windings are being wound. There are many different indirect designs and this is just one of them. All indirect magnetic switch models consist of additional cores for the control windings which have common sections with the main gapped ferrite core. Dose linked but separate input and output magnetic circuits, allow for differentiation between the input and output parameters and more flexible design of the device. Some of the indirect control models theoretically seem not to be working but others seem to have potential due to proper separation between the input and output magnetic fluxes. 


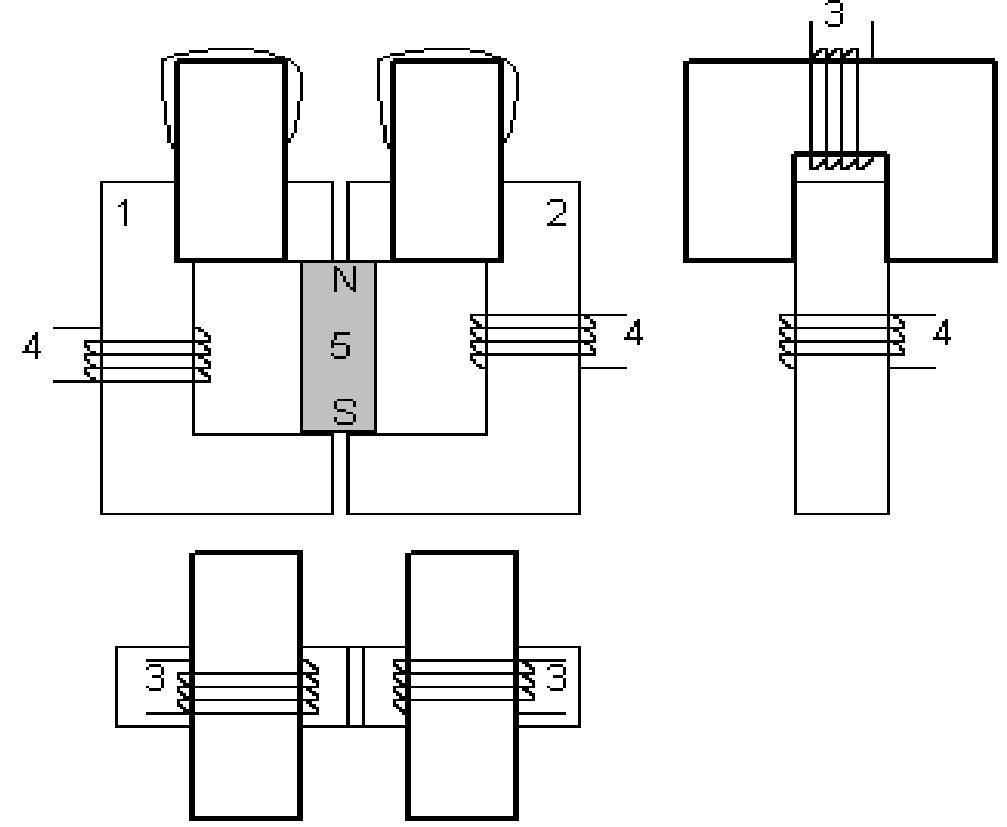

Fig 7 Indirect control: 1 and 2- ferrite core halves; 3- control windings; 4 - output windings; 5 - permanent magnet

While the magnetic permeability of both pathways 1 and 2 are equal the permanent magnet flux should be separated equally between them. When power is applied to one of the control windings, its core gets magnetized and this will create an opposing magnetic force in the respective common section, which is supposed to lead to redistribution of the permanent magnet field. Theoretically the redirection should happen only if the created magneto-motive force is at least a couple of times stronger than that of the permanent magnet one [16]. When the second control winding gets activated the process develops in opposite direction.

The main idea of control is the whole magnetic flux created by the energy consumed by any of the control windings to be closed in their own ferrite core. If this is achieved, then if any of the control windings is powered up this won't induce voltages in the others. In this case the input windings with their ferrite cores should work as solenoids which when powered up will store certain amount of energy and when power is disconnected they will give back almost the same quantity. During this process if part of the permanent magnet field flowing in the main gapped ferrite core with halves 1 and 2, gets redirected then voltage will be induced in the output windings 4 . How much energy will be given at the output windings depends on the achieved level of permanent magnet flux redirection. Whether different magnetic switches designs work or not is a matter of future research. Theoretically the indirect way of control of magnetic flux seems to have potential and under some special design conditions it seems to be possible a working model to be created.

\section{CONCLUSION}

If a magnet is incorporated into a magnetic system and if there are two equal high-permeability pathways then the magnetic force lines redistribute evenly between them.
How much the core gets magnetized is easy to calculate when the permanent magnet parameters and the ferrite core parameters are known when the air-gap is comparatively small. The bigger the airgap, the lower the measured core flux density which is due to the fact that more of the magnetic force lines flow trough the space instead of trough the ferrite core. The bigger the airgap, he bigger the error of calculation, but it could be corrected.

It is pretty hard to find different magnets that much with certain ferrite core size which makes it difficult to extend the scope of this research.

Incorporation of a permanent magnet leads to slight lowering of the induction compared to the case without it. The permanent magnet field partially magnetizes the core which should be taken under consideration during the design process.

Redirection of the flow of magnetic force lines of permanent magnet between two high-permeability path ways by change of their air-gap lengths respectively their magnetic permeabilities is possible. This redirection leads to change of the magnetic flux density in both pathways which induces electromotive force in the output windings. The higher and faster the air-gap lengths change the higher the induced voltages.

Neodymium magnets are said to have lifespan between 10 to 20 years. If they work in an environment with strong magnetic fields their lifespan could be shortened. Guiding the static magnetic flux to flow in one or another direction shouldn't be considered as worst case scenario as guiding the static magnetic field doesn't means opposing it. 
Patent research revealed that there are designs of magnetic flux switches dating from 1900 to 2010. Most of the magnetic switches designs are working based on creation of opposing magnetomotive force or magnetic permeability change of one of usually two possible high permeability pathways. Most of motionless ways of redirection of the permanent magnet flux that has been found uses some type of control windings.

Based on the theory the direct way of control of strong magnetic field with low drive power seems to be impossible, yet there are patents claiming that working models have been developed. Often patent holders omit important details about their inventions with the clear aim others not to be able to recreate them.

Theoretically the indirect way of control of magnetic flux seems to have potential and under some special design conditions it seems to be possible a working model to be created.

\section{REFERENCES}

[1]. H. Knoepfel, Magnetic Fields, John Wiley \& Sons, 2000.

[2]. R. O'Handley, Modern Magnetic Materials, John Wiley \& Sons, 2000.

[3]. Wm. McLyman, Transformer and Inductor Design, Magnetics Inc, USA 2004.

[4]. D.Fleisch, A Student's Guide to Maxwell's Equations, Cambridge University Press, 2008.

[5]. J. Kraus, K. Carver, Electromagnetics, 2nd ed., McGraw Hill, 1982

[6]. R. L. Rauck, Magnetic Flux Density Equation Revision.

[7]. A.V. Bossche, V. Valchev, Inductors and Transformers for Power Electronics, Ghent University, 2005.

[8]. Flynn,C.J., Methods for controlling the path of magnetic flux from a permanent magnet and devices incorporating the same. US Patent No: US 6,246,561 B1, 2001.

[9]. Patrick, S., T. Bearden, J. Hayes, K. Moore, J. Kenny. Motionless electromagnetic generator. US Patent No: US 6,362,718, 2002.

[10]. Annis, T., P. Eberly. Energy generation apparatus and methods based upon magnetic flux switching. US Patent App. 20090096219, 2009.

[11]. Davidson, D., Acoustic -magnetic power generator. US Patent 5,568,005, 1996

[12]. Barbat, W., Self-sustaining electric-power generator utilizing electrons of low inertial mass to magnify inductive energy. US Patent App. 20070007844 A1, 2007

[13]. Willis, R., Electrical Generator. US Patent App. WO 2009 065210(A1), 2009

[14]. Gunderson, G., Solid-State Electric Generator. US Patent App. 2006/0163971 A1, 2006

[15]. Abele, M., Structures of permanent magnets, generation of uniform fields, John Wiley\& Sons Inc., NY

[16]. Moskowitz, L. Permanent Magnet Design and Application Handbook. Krieger Publishing Company 1995

[17]. Ueno, T., T. Higuchi. Novel composite of magnetostrictive material and piezoelectric actuator for coilfree magnetic force control, Japan, Elsevier 2005
[18]. Radus, R. Permanent-Magnet Circuit using a FluxTransfer Principle, Engineers' Digest, 1963

[19]. Radus, R. Fail Safe Electromagnetic Lifting Device. US Patent No: US 3,316,514, 1967

[20]. Kaganov, M., V. Tsukernik. The Nature of Magnetism, Russia, Mir Publishers, 1985

[21]. Rivas, E., Electromagnetic generator, US patent 4,006,401, 1977

[22]. Keseev, V. An opportunity for development of new generation power supplies - in bulgarian , Ruse University, Ruse, 2013

"The present document has been produced with the financial assistance of the European Social Fund under Operational Programme "Human Resources Development". The contents of this document are the sole responsibility of the "Angel Kanchev" University of Ruse and can under no circumstances be regarded as reflecting the position of the European Union or the Ministry of Education and Science of Republic of Bulgaria."

Project № BG051PO001-3.3.06-0008 “Supporting Academic Development of Scientific Personnel in Engineering and Information Science and Technologies". 